\title{
Extracción asistida con ultrasonido de compuestos fenólicos de dos variedades de papas (Solanum phureja) nativas andinas y evaluación de su actividad antioxidante
}

\author{
Roxana Benavides-Guerrero*, Yudy A. Revelo-Cuarán, Oscar Arango-Bedoya y Oswaldo Osorio-Mora \\ Facultad de Ingeniería Agroindustrial, Universidad de Nariño. San Juan de Pasto, Colombia. \\ (correo-e: roxanabegue@gmail.com; andrea.revelo94@gmail.com; oscar.arangob@gmail.com; \\ osorio_oswaldo@udenar.edu.co) \\ * Autor a quien debe ser dirigida la correspondencia.
}

Recibido Ene. 2, 2020; Aceptado Mar. 5, 2020; Versión final Jun. 6, 2020, Publicado Oct. 2020

\begin{abstract}
Resumen
El objetivo de este estudio fue evaluar el contenido de fenoles totales (FT) y actividad antioxidante de dos variedades de papas (Solanum phujera) nativas cultivadas al sur de Colombia. Las papas nativas tienen un gran potencial debido a su alto contenido de compuestos bioactivos. Se realizaron dos diseños experimentales, el primero para determinar el mejor solvente para extraer los FT y el segundo para evaluar el efecto del uso de ultrasonido en la extracción de FT. La actividad antioxidante se evaluó por los métodos de ABTS $^{+}$y DPPH. El mejor solvente fue acetona al $70 \%$ (v/v). La utilización de ultrasonido aumentó $118-195$ $\%$ la extracción de FT. El contenido de FT fue mayor en la piel que en la pulpa. Se concluye que las variedades de $S$. phujera estudiadas son una buena fuente de compuestos antioxidantes, pero se requiere identificar y cuantificar los compuestos presentes, tales como antocianinas.
\end{abstract}

Palabras clave: papas nativas; fenoles totales; actividad antioxidante; extracción asistida por ultrasonido; antioxidantes

\section{Ultrasound-assisted extraction of phenolic compounds from two varieties of an Andean native potato (Solanum phureja) and evaluation of their antioxidant activity}

\begin{abstract}
The objective of this research study was to evaluate the content of total phenols (TP) and the antioxidant activity of two varieties of native potatoes (Solanum phujera) grown in southern Colombia. The varieties of native potatoes have a great potential due to their high content of bioactive compounds. Two experimental designs were carried out, the first to determine the best solvent to extract TP and the second to evaluate the effect of using ultrasound in TP extraction. The antioxidant activity was evaluated by the ABTS ${ }^{+}$and DPPH methods. The best solvent was $70 \%$ acetone $(\mathrm{v} / \mathrm{v})$ and the application of ultrasound increased TP extraction by 118 to $195 \%$. TP content was greater in the peel than in the flesh. It is concluded that the examined $S$. phujera varieties are good sources of antioxidant compounds, but the presence of compounds such as anthocyanins requires further identification and quantification.
\end{abstract}

Keywords: native potatoes; total phenols; antioxidant activity; ultrasound-assisted extraction; antioxidants 


\section{INTRODUCCIÓN}

Las papas nativas (Solanum tuberosum ssp. andigena y Solanum phureja) son producto de la selección y domesticación llevada a cabo durante décadas por comunidades indígenas y campesinas principalmente en la zona de los Andes sudamericanos (Segura, 2014). Estas variedades representan actualmente un gran banco de germoplasma por su resistencia a plagas y enfermedades, fácil adaptación y tolerancia a factores climáticos (Martínez, 2015). Las papas nativas poseen un elevado contenido de materia seca, bajo nivel de azúcares reductores, excelente calidad culinaria y un contenido nutricional superior al de las variedades comerciales (Segura, 2014). Muchas de estas variedades presentan pigmentaciones rojas, moradas y amarillas en cáscara y pulpa, proporcionando una fuente natural de compuestos fitoquímicos como carotenoides, antocianinas y otros compuestos fenólicos (Reyes et al., 2005), los cuales son reconocidos por sus propiedades antioxidantes, antiinflamatorias y antimicrobianas.

La papa por ser un cultivo ampliamente producido y consumido a nivel mundial, podría constituir una fuente ideal de antioxidantes para la dieta humana. Una significativa actividad antioxidante en tubérculos de papa ha sido extensamente reportada (Reddivari et al., 2007). Se ha demostrado que los genotipos de papa pigmentada contienen niveles más altos de actividad antioxidante; especialmente cultivos con pulpa púrpura y roja comparados con los de pulpa blanca y amarilla (Wegener et al., 2009). Cerón-Lasso et al. (2018) estudiaron la composición fisicoquímica de 21 genotipos de papas criollas (Solanum tuberosum Phureja) observando que los genotipos con cáscara de color rojo o morado presentaron tasas de recuperación de fenoles totales 1.65 - 2.74 veces más altos comparado con los genotipos de color amarillo.

Adicionalmente André et al. (2009) encontraron que las variedades de papas nativas andinas cultivadas en diversas condiciones ambientales presentan una alta estabilidad en el contenido de compuestos fenólicos, por lo que podrían usarse en programas de mejoramiento genético para mejorar el contenido de compuestos benéficos para la salud. La determinación del contenido de compuestos fenólicos y su actividad antioxidante se ve afectada por las variables que intervienen en el proceso de extracción, tales como la naturaleza química de la muestra, el método de extracción, el solvente usado, daño de compuestos termolábiles y la presencia de sustancias interferentes, por lo que actualmente se investiga la utilización de técnicas más favorables y amigables con el medio ambiente (Michiels et al., 2012). Algunos estudios han demostrado que la aplicación de ultrasonido en la extracción de compuestos de matrices vegetales puede incrementar la obtención y reducir el tiempo y el consumo de solvente, siendo los factores que intervienen la frecuencia de la onda, el poder de sonificación, el tiempo y la distribución de la onda ultrasónica (Sharayei et al., 2019).

El procesamiento de la papa genera grandes cantidades de subproductos, que incluyen la cáscara y partes de la pulpa. Riciputi et al. (2018) extrajeron compuestos fenólicos de subproductos de la industria de fritura de cinco variedades de papa, optimizando las condiciones de extracción con un baño ultrasónico, encontrando que el ácido clorogénico representaba el $49.3-61 \%$ del total de estos compuestos y una alta correlación con la actividad antioxidante, se observó que la extracción asistida con ultrasonido (EAU) mejoró el rendimiento de la extracción de compuestos bioactivos del interior de la matriz vegetal, debido a los efectos de disrupción celular y cavitación que mejoran el contacto soluto/solvente. Por otra parte, Tutunchi et al. (2019), encontraron que el uso de ultrasonido redujo la degradación de los compuestos fenólicos extraídos de remolacha (Beta vulgaris $L$.). Esta tecnología emergente puede abrir nuevos horizontes para la extracción de compuestos bioactivos y su aplicación en los campos alimentario, nutracéutico y médico. En este contexto, el objetivo de este trabajo fue determinar el contenido de compuestos fenólicos y la actividad antioxidante de extractos obtenidos por ultrasonido de dos variedades de papa nativa cultivadas en la zona andina del suroccidente de Colombia.

\section{METODOLOGÍA}

Para esta investigación se utilizaron papas nativas (Solanum phureja) de las variedades ratona morada (RM) y curiquinga (CQ) (Fig. 1). Los materiales se recolectaron en parcelas experimentales pertenecientes al Centro de Investigación Obonuco de la Corporación Colombiana de Investigación Agropecuaria (CORPOICA), en el mes de febrero de 2018. Las parcelas están ubicadas en el municipio de Pasto ( $\mathrm{N} 01^{\circ} 11^{\prime} 4.13^{\prime \prime} \mathrm{W} 77^{\circ}$ $\left.19^{\prime} 0.19^{\prime \prime}\right)$, suroccidente de Colombia, a 2905 m.s.n.m., temperatura media anual $13.8^{\circ} \mathrm{C}$ y precipitación anual promedio de $982.7 \mathrm{~mm}$.

El material vegetal se seleccionó según su sanidad, se lavó con agua potable y se peló manualmente. La pulpa se cortó en cubos de aproximadamente $1 \mathrm{~cm}$ de lado. Las muestras de cáscara y pulpa de ambas variedades se congelaron en un ultracongelador (Menhen MB12T, Dartico, Bogotá) a $-30^{\circ} \mathrm{C}$ por $2 \mathrm{~h}$, luego se secaron en un liofilizador (Scientz - 18ND) por $25 \mathrm{~h}$ hasta una humedad final de $4 \%$. Las muestras secas se molieron con un molino de cuchillas (IKA A11 Basic) y se tamizaron, seleccionando un tamaño de partícula de $0.228 \mathrm{~mm}$. El material en polvo se empacó en bolsas multicapa que protege de la luz, la humedad y la transferencia de gases, se sellaron a vacío y se almacenaron a $4{ }^{\circ} \mathrm{C}$ hasta su uso. 

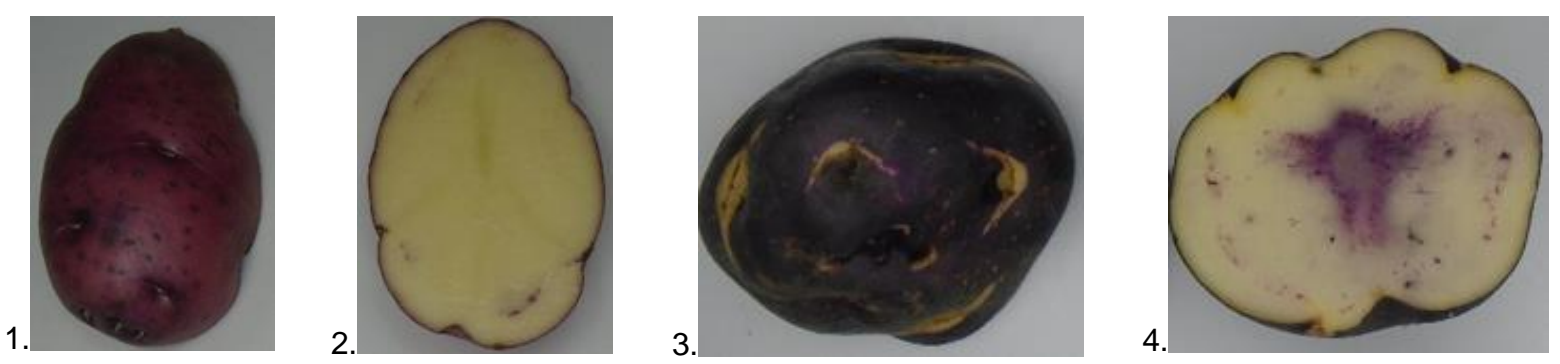

Fig. 1: Papas nativas estudiadas: imágenes 1 y 2 variedad Ratona Morada $(\mathrm{RM})$, imágenes 3 y 4 variedad Curiquinga (CQ)

\section{Análisis químico proximal de las muestras}

Se utilizaron muestras frescas de ambos materiales de papa, se analizaron pulpa y cáscara en conjunto para la determinación de humedad, materia seca, cenizas, extracto etéreo, fibra cruda, proteína y calcio, utilizando métodos oficiales de análisis de la AOAC (2000).

\section{Extracción convencional de compuestos fenólicos (EC)}

Se llevó a cabo un diseño factorial completamente aleatorizado con el fin de determinar el solvente más apropiado para la extracción de compuestos fenólicos, y usarlo en la extracción asistida con ultrasonido. Con base en revisión de literatura (Cuesta-Parra y Correa-Mahecha, 2018; Guntero et al., 2015), se seleccionaron como solventes metanol al $70 \%(\mathrm{v} / \mathrm{v})$ (metanol-agua) y acetona al $70 \%(\mathrm{v} / \mathrm{v})$ (acetona - agua) y se realizaron extracciones convencionales sólido - líquido (maceración) de cáscara y pulpa por separado para ambas variedades de papa (Moreno, Ortiz, y Restrepo, 2014). Se prepararon soluciones al $5 \%$ (p/v) de cada muestra en los solventes seleccionados y se dejaron en agitación a temperatura ambiente por 15 min (Guntero et al., 2015). Los extractos fueron centrifugados por $10 \mathrm{~min}$ a $10000 \mathrm{rpm} \mathrm{a} 4^{\circ} \mathrm{C}$, los sobrenadantes fueron filtrados inmediatamente, envasados en viales ámbar y almacenados en refrigeración hasta su posterior análisis. Como variable de respuesta se evaluó el contenido de fenoles totales en los extractos.

\section{Extracción asistida por ultrasonido de compuestos fenólicos (EAU)}

Se prepararon soluciones al $5 \%(\mathrm{p} / \mathrm{v})$ muestra - solvente usando el solvente óptimo determinado en la extracción convencional. Se realizó la extracción de compuestos fenólicos de la cáscara y la pulpa (por separado) de ambas variedades de papa usando un baño ultrasónico (Fisherbrand FB11205, Thermo Fisher Scientific). Se utilizó un diseño factorial completamente aleatorizado para evaluar el efecto del tiempo de extracción (10, 30 y $50 \mathrm{~min})$, la temperatura del baño $\left(30\right.$ y $\left.50^{\circ} \mathrm{C}\right)$ y la frecuencia de ultrasonido $(37 \mathrm{y} 80 \mathrm{kHz})$ sobre el contenido de fenoles totales en los extractos. La potencia del equipo se fijó en $154 \mathrm{~W}$. Los extractos fueron centrifugados por $10 \mathrm{~min}$ a $10000 \mathrm{rpm}$ a $4^{\circ} \mathrm{C}$ y almacenados en refrigeración hasta su análisis. El diseño experimental se realizó por triplicado para un total de 144 ensayos.

\section{Determinación del contenido de fenoles totales}

Se determinó usando el método de Folin - Ciocalteu, de acuerdo a la metodología de Kuskoski et al., (2005), con algunas modificaciones (Moreno et al., 2014). Para la reacción se utilizaron $0.1 \mathrm{~mL}$ de extracto, $0.5 \mathrm{~mL}$ del reactivo de Folin - Ciocalteu y $1.5 \mathrm{~mL}$ de solución acuosa de $\mathrm{Na}_{2} \mathrm{CO}_{3}$ al $10 \%$. La solución se dejó en un lugar oscuro y transcurridas dos horas de reacción se tomó la lectura de absorbancia a $765 \mathrm{~nm}$ en un espectrofotómetro (Genesis 10UV-Vis Scanning, Thermo Fisher Scientific). Se elaboró una curva de calibración con un patrón de ácido gálico empleando soluciones de 25, 50, 100, 150, 250 y 500 ppm para expresar los resultados en $\mathrm{mg}$ de ácido gálico equivalente (AGE)/100 g de muestra seca.

\section{Evaluación de la actividad antioxidante}

Se evaluó la actividad antioxidante de los extractos con mayor contenido de fenoles totales usando los métodos de $\mathrm{ABTS}^{+}$y DPPH. Para el método de $\mathrm{ABTS}^{+}$se usó la metodología descrita por Re et al., (1999), donde se mezclaron $30 \mu \mathrm{L}$ del extracto vegetal y $3 \mathrm{~mL}$ de solución ABTS•+, se agitó y después de 10 min se tomó lectura de absorbancia. Se realizó una curva de calibración con Trolox a diferentes concentraciones $(0.5$ - $2.5 \mathrm{mM}$ ), los resultados se expresaron en equivalentes de Trolox (TEAC; mmol de Trolox/ g de muestra seca). Para el método de DPPH'se siguió el procedimiento de Brand-Williams et al., (1995), para la reacción de la muestra se tomaron $2925 \mu \mathrm{L}$ de la solución del radical, se adicionaron $75 \mu \mathrm{L}$ del extracto de papa y se registró la absorbancia en intervalos de $10 \mathrm{~s}$ hasta un estado estacionario. Se elaboró una curva de calibración con diferentes disoluciones de DPPH• $(0.0025-0.050 \mathrm{mM})$ y según Sánchez-Moreno et al., (1998) 
determinaron los parámetros $\mathrm{EC}_{50}$, el cual hace referencia a la concentración de antioxidante necesario para reducir la concentración inicial del radical a la mitad y tec50, el tiempo necesario para alcanzar la $\mathrm{EC}_{50}$. Los dos parámetros anteriores se pueden relacionar y se obtiene un parámetro más adecuado, la eficiencia anti radical $(E A)$, que se calcula como el inverso del producto de $E_{50} \times t_{E C 50}$, cuanto más bajo es $E_{50}$, más bajo es el teC50 y más alta es la EA.

\section{Contenido de ácido clorogénico}

Se determinó el contenido de ácido clorogénico en cáscara y pulpa de ambas variedades de papa de acuerdo a la metodología propuesta por Ola et al., (2009), mediante cromatografía líquida de alta resolución. La identificación de los extractos metanólicos se realizó mediante comparación con los tiempos de retención de una solución estándar (Sigma-Aldrich, USA) y mediante análisis de los espectros UV-VIS. La cuantificación se realizó empleando estándar externo y relaciones de área cromatográfica con soluciones patrón de ácido clorogénico.

\section{Análisis estadístico}

El análisis de datos se realizó con el programa Statgraphics Centurion@ versión XVI. Se realizaron análisis de varianza para determinar diferencias significativas entre tratamientos. El criterio para decidir la significancia estadística fue $\mathrm{p}<0.05$. La comparación de medias se realizó por el método de diferencia significativa mínima de Fisher (LSD) con $5 \%$ de nivel de significancia. Los datos presentados en tablas muestran las medias y desviaciones estándar de 3 réplicas y letras distintas indicas diferencias significativas.

\section{RESULTADOS Y DISCUSIÓN}

A continuación, se describen los resultados obtenidos y el análisis de estos respecto a la eficiencia de la EAU para la extracción de fenoles totales y la cuantificación de éstos en las variedades de papa nativa estudiadas, así como su capacidad antioxidante.

\section{Análisis proximal}

En la Tabla 1 se muestra la composición proximal para ambas variedades de papa nativa. Se puede destacar el valor nutricional de las papas nativas, debido a su contenido de vitaminas, minerales, fibra, aminoácidos esenciales y compuestos con potencial antioxidante como el ácido ascórbico, $\alpha$-tocoferol, carotenoides, diferentes polifenoles y ácidos fenólicos (Solarte-Montúfar et al., 2019). El análisis de varianza mostró que solo hubo diferencias significativas $(p<0.05)$ en los contenidos de fibra y calcio entre ambas variedades, lo que se puede atribuir a factores genotípicos relacionados con la variedad, condiciones agroecológicas y labores del cultivo (Villacrés, Quilca, Muñoz, Monteros, \& Reinoso, 2009).

Tabla 1: Análisis químico proximal de las dos variedades de papa nativa ( $\mathrm{RM}$, variedad ratona morada; $\mathrm{CQ}$, variedad curiquinga)

\begin{tabular}{|l|l|l|}
\hline \multirow{2}{*}{ Parámetro } & Variedad \\
\cline { 2 - 3 } & $R M$ & $C Q$ \\
\hline Humedad $(\mathrm{g} / 100 \mathrm{~g})$ & $81.00 \pm 0.06^{\mathrm{a}}$ & $82.85 \pm 0.05^{\mathrm{a}}$ \\
\hline Cenizas $(\mathrm{g} / 100 \mathrm{~g})$ & $1.10 \pm 0.03^{\mathrm{a}}$ & $1.00 \pm 0.03^{\mathrm{a}}$ \\
\hline Fibra cruda $(\mathrm{g} / 100 \mathrm{~g})$ & $0.04 \pm 0.003^{\mathrm{a}}$ & $0.50 \pm 0.002^{\mathrm{b}}$ \\
\hline Extracto no nitrogenado $(\mathrm{g} / 100 \mathrm{~g})$ & $15.81 \pm 1.07^{\mathrm{a}}$ & $14.13 \pm 1.04^{\mathrm{a}}$ \\
\hline Proteína $(\mathrm{g} / 100 \mathrm{~g})$ & $1.96 \pm 0.01^{\mathrm{a}}$ & $1.46 \pm 0.01^{\mathrm{a}}$ \\
\hline Calcio $(\mathrm{mg} / 100 \mathrm{~g})$ & $26.13 \pm 2.65^{\mathrm{a}}$ & $12.90 \pm 2.86^{\mathrm{b}}$ \\
\hline
\end{tabular}

\section{Extracción convencional de fenoles totales}

Se extrajeron por maceración los FT de cáscara y pulpa (por separado) de las dos variedades de papa nativa usando como solventes metanol y acetona, ambos al $70 \%(\mathrm{v} / \mathrm{v})$, con el fin de elegir el solvente que permite una mejor extracción de compuestos fenólicos. Los valores obtenidos en la determinación de FT se muestran en la Tabla 2. Con excepción de la cáscara de la variedad RM, el contenido de FT en los extractos obtenidos con acetona fue significativamente más alto que en aquellos obtenidos con metanol. El contenido de FT fue significativamente más alto en la variedad $\mathrm{CQ}$, debido posiblemente a que esta presenta coloración púrpura no sólo en cáscara sino también en pulpa, lo que se relaciona con altos contenidos de antocianinas y flavonoides (Lachman et al., 2008). 
Los resultados de este estudio coinciden con los de Do et al., (2014), quienes encontraron que el rendimiento de extracción de polifenoles con acetona al $50 \%$ fue mayor que el obtenido con metanol al 50 y al $75 \%$. Boeing et al., (2014) también observaron que una solución acuosa de acetona al 70 \% fue la más eficiente, entre una amplia variedad de solventes, para la extracción de compuestos fenólicos de diferentes bayas. La polaridad del disolvente desempeña un papel clave en el aumento de la solubilidad de fenoles; en general, se ha encontrado que el metanol es más eficiente en la extracción de polifenoles de peso molecular más bajo, mientras que la acetona acuosa es buena para la extracción de flavonas de peso molecular más alto (Dai y Mumper, 2010).

Tabla 2: Fenoles totales en los extractos obtenidos por extracción convencional. ( $R M$, variedad ratona morada; $C Q$, variedad curiquinga)

\begin{tabular}{|c|c|c|c|}
\hline Solvente & Variedad & Material & $\begin{array}{l}\text { Fenoles Totales } \\
\text { (mg AGE/100 g bs) }\end{array}$ \\
\hline \multirow[t]{2}{*}{ Acetona } & $\mathrm{RM}$ & $\begin{array}{l}\text { Cáscara } \\
\text { Pulpa }\end{array}$ & $\begin{array}{l}650.68 \pm 25.93^{d} \\
324.15 \pm 12.13^{b}\end{array}$ \\
\hline & $\mathrm{CQ}$ & $\begin{array}{l}\text { Cáscara } \\
\text { Pulpa }\end{array}$ & $\begin{array}{l}877.67 \pm 50.21^{f} \\
448.26 \pm 20.03^{c}\end{array}$ \\
\hline \multirow[t]{2}{*}{ Metanol } & $\mathrm{RM}$ & $\begin{array}{l}\text { Cáscara } \\
\text { Pulpa }\end{array}$ & $\begin{array}{l}628.89 \pm 24.52^{\mathrm{d}} \\
275.90 \pm 2.71^{\mathrm{a}}\end{array}$ \\
\hline & $\mathrm{CQ}$ & $\begin{array}{l}\text { Cáscara } \\
\text { Pulpa }\end{array}$ & $\begin{array}{l}802.94 \pm 40.36^{\mathrm{e}} \\
330.77 \pm 15.11^{\mathrm{b}}\end{array}$ \\
\hline
\end{tabular}

\section{Efecto de las condiciones de EAU}

La Tabla 3 muestra los resultados del análisis de varianza (ANOVA), de la EAU de FT en cáscara y pulpa de las dos variedades de papa nativa, usando como solvente acetona al $70 \%(\mathrm{v} / \mathrm{v})$. Se observa que todos los factores experimentales y sus interacciones tuvieron efecto significativo sobre el contenido de FT en los extractos.

Tabla 3: Análisis de varianza de la EAU de fenoles totales en cáscara y pulpa de dos variedades de papas nativas

\begin{tabular}{|l|l|l|l|l|l|}
\hline Fuente & Suma de cuadrados & Gl & Cuadrado Medio & Razón $-F$ & $p$-valor \\
\hline A: Frecuencia & $2.66384 \mathrm{E} 6$ & 1 & $2.66384 \mathrm{E} 6$ & 323.12 & 0.0000 \\
\hline B: Material & $5.9432 \mathrm{E} 6$ & 3 & $1.98107 \mathrm{E} 6$ & 240.30 & 0.0000 \\
\hline C: Temperatura & $2.32131 \mathrm{E} 6$ & 1 & $2.32131 \mathrm{E} 6$ & 281.57 & 0.0000 \\
\hline D: Tiempo & $3.3574 \mathrm{E} 6$ & 2 & $1.6787 \mathrm{E} 6$ & 203.63 & 0.0000 \\
\hline AB & 116195 & 3 & 38731.8 & 4.70 & 0.0039 \\
\hline AC & 350290 & 1 & 350290 & 42.49 & 0.0000 \\
\hline AD & 457735 & 2 & 228868 & 27.76 & 0.0000 \\
\hline BC & 114729 & 3 & 38242.9 & 4.64 & 0.0042 \\
\hline BD & 211605 & 6 & 35267.5 & 4.28 & 0.0006 \\
\hline CD & 633200 & 2 & 316600 & 38.40 & 0.0000 \\
\hline Residuos & 981038 & 119 & 8244.01 & & \\
\hline Total (corr.) & $1.71505 E 7$ & 143 & & & \\
\hline
\end{tabular}

En la tabla 4 se observan las diferencias entre las medias del contenido de FT en los extractos obtenidos con cada uno de los niveles de los factores evaluados en la EAU. En la Tabla, CRM es cáscara ratona morada; CCQ es cáscara curiquinga; PRM es pulpa ratona morada; y PCQ es pulpa curiquinga. Cada valor es la media de tres determinaciones. Letras diferentes en cada columna indican diferencias significativas $(p<0.05)$.

Tabla 4: Medias de fenoles totales obtenidos en los diferentes tratamientos

\begin{tabular}{|l|l|l|l|l|l|l|l|}
\hline Material & Media & Tiempo $(\mathrm{min})$ & Media & Frecuencia $(\mathrm{kHz})$ & Media & Temperatura $\left({ }^{\circ} \mathrm{C}\right)$ & Media \\
\hline CRM & $845.33^{\mathrm{b}}$ & 10 & $559.65^{\mathrm{a}}$ & 37 & $869.55^{\mathrm{b}}$ & 30 & $606.57^{\mathrm{a}}$ \\
\hline CCQ & $1010.07^{\mathrm{c}}$ & 30 & $709.59^{\mathrm{b}}$ & 80 & $597.53^{\mathrm{a}}$ & 50 & $860.50^{\mathrm{b}}$ \\
\hline PRM & $520.32^{\mathrm{a}}$ & 50 & $931.37^{\mathrm{c}}$ & - & - & - & - \\
\hline PCQ & $558.43^{\mathrm{a}}$ & - & - & - & - & - & - \\
\hline
\end{tabular}


Al comparar las tablas 3 y 4 se puede observar que el contenido de fenoles totales extraído con acetona de la cáscara aumentó $172 \%$ y $118 \%$ para las variedades ratona morada y curiquinga respectivamente mediante el uso de EAU, mientras que para el caso de la pulpa el aumento fue de 167 y $195 \%$. Sharayei et al., (2019) argumentan que el ultrasonido es una técnica que aumenta significativamente la extracción, sin degradación de carotenoides en comparación con otras técnicas convencionales, además, sería de gran importancia para el aprovechamiento de subproductos como cáscaras, semillas y partes de pulpa de materiales vegetales.

En el proceso de EAU la extracción de FT se incrementó con el tiempo y la temperatura y disminuyó al aumentar la frecuencia. Los contenidos más altos de FT tanto en cáscara como en pulpa, se obtuvieron a 50 $\min , 50^{\circ} \mathrm{C}$ y $37 \mathrm{kHz}$, mientras que los más bajos fueron a $10 \mathrm{~min}, 30^{\circ} \mathrm{C}$ y $80 \mathrm{kHz}$ (Tabla 4), coincidiendo con los resultados de Kumari et al., (2017). En general, se han identificado varios mecanismos implicados en la EAU. La fragmentación debida a la colisión entre partículas y ondas ultrasónicas provoca una reducción en el tamaño de las partículas, facilitando así la transferencia de masa. También la sono-capilaridad y la sonoporación pueden mejorar la penetración del líquido a través de los canales producidos por la implosión de la burbuja y la alteración de la permeabilidad de las membranas celulares. La eficiencia de la EAU puede verse afectada por distintas variables, como las propiedades físicas del solvente, temperatura, amplitud de onda, frecuencia, potencia del ultrasonido, presión hidrostática, tensión superficial, potencia de irradiación, pH disolvente y la relación disolvente - sólido, por otra parte la cavitación causa ciclos de expansión y contracción en el material vegetal, causando una interrupción en las paredes celulares de la matriz sólida, aumentando así la extracción (Campo-Vera, Gélvez-Ordoñez, y Ayala-Aponte, 2018).

En este estudio el aumento de la frecuencia de ultrasonido de 37 a $80 \mathrm{kHz}$ ejerció un efecto negativo sobre la eficiencia de extracción de fenoles totales, coincidiendo con lo observado por Kumari et al., (2017), evaluaron en cáscara de dos variedades de papa el efecto de la frecuencia/potencia ultrasónica de $33 \mathrm{kHz}(100 \mathrm{~W})$ y 42 $\mathrm{kHz}(45 \mathrm{~W})$, encontrando que la frecuencia más baja (con mayor potencia de salida) fue más efectiva en la extracción de fenoles. La intensidad de cavitación está inversamente relacionada con la frecuencia ultrasónica, por lo que al generar burbujas cavitacionales más grandes, pero relativamente menores, implosionan con una mayor energía y da lugar a un mayor grado de ruptura celular (Campo-Vera et al., 2018).

\section{Actividad antioxidante de los extractos fenólicos}

Los resultados de la interacción entre los extractos obtenidos por EAU y los radicales libres indicaron una buena capacidad de inhibición de radicales, lo cual sugiere que en las muestras analizadas se encuentran compuestos donadores de hidrogeno o electrones que pueden estabilizar la reacción con los radicales ABTS ${ }^{+}$ y DPPH• (Tabla 5). En la Tabla 5, CRM es cáscara ratona morada; CCQ es cáscara curiquinga; PRM es pulpa ratona morada; y PCQ es pulpa curiquinga. Cada valor es la media de tres determinaciones. Letras diferentes en cada columna indican diferencias significativas $(p<0.05)$. El análisis de varianza indicó que no hubo diferencias en la actividad antioxidante de los extractos obtenidos de la cáscara de ambas variedades de papa evaluada por el método de $\mathrm{ABTS}^{+}$, sin embargo, en la evaluación por DPPH se observó que la eficiencia anti radical (EA) del extracto de la cáscara de la variedad $C Q$ fue mayor, lo que coincide con su mayor contenido de FT. En el caso de la pulpa, la actividad antioxidante evaluada por los dos métodos fue significativamente mayor en la variedad CQ.

Tabla 5: Actividad antioxidante de los extractos obtenidos mediante EAU

\begin{tabular}{|c|c|c|c|c|c|c|}
\hline \multirow[t]{2}{*}{ Extracto } & \multirow{2}{*}{$\begin{array}{l}\text { Fenoles totales } \\
\left(m g \text { AGE } 100 \mathrm{~g} \mathrm{bs}^{-1}\right)\end{array}$} & \multirow{2}{*}{$\begin{array}{l}\text { Ácido clorogénico } \\
\left(m g g b s^{-1}\right)\end{array}$} & \multirow{2}{*}{$\begin{array}{l}\text { ABTS }^{+} \\
\left(m M \text { Trolox } g b s^{-1}\right)\end{array}$} & \multicolumn{3}{|l|}{$D P P H$} \\
\hline & & & & $E C_{50}$ & $t_{E C 50}(\mathrm{~min})$ & $E A \times 10^{-3}$ \\
\hline CRM & $1770.25 \pm 27.13^{c}$ & $0.38^{c}$ & $0.410 \pm 0.002^{c}$ & $4.51^{b}$ & $5.57^{\mathrm{b}}$ & $39.81^{c}$ \\
\hline $\mathrm{CCQ}$ & $1910.42 \pm 20.72^{d}$ & $0.44^{d}$ & $0.412 \pm 0.002^{c}$ & $4.05^{\mathrm{a}}$ & $4.94^{\mathrm{a}}$ & $49.98^{d}$ \\
\hline PRM & $865.3 \pm 60.46^{a}$ & $0.06^{a}$ & $0.303 \pm 0.005^{\mathrm{a}}$ & $22.59^{d}$ & $6.83^{d}$ & $6.48^{\mathrm{a}}$ \\
\hline$P C Q$ & $1324.11 \pm 23.50^{b}$ & $0.13^{\mathrm{b}}$ & $0.324 \pm 0.007^{b}$ & $21.78^{c}$ & $6.00^{c}$ & $7.65^{\mathrm{b}}$ \\
\hline
\end{tabular}

Según el estudio realizado por Al-Weshahy et al., (2013), la actividad antioxidante evaluada por $\mathrm{ABTS}^{+}$de los extractos de cáscara de seis variedades de papas con pigmentación roja, obtenidos mediante extracción convencional y usando metanol como solvente, fue de $0.28-0.55 \mathrm{mM}$ de Trolox gbs ${ }^{-1}$, estos resultados son similares a los encontrados en esta investigación, confirmando que los polifenoles en papas nativas se encuentran ubicados en mayor cantidad en la cáscara (Araya et al., 2006). Según Sánchez-Moreno et al., (1998), el comportamiento cinético de una solución antioxidante puede clasificarse de la siguiente manera: "rápido" $t_{E C 50}<$ a $5 \mathrm{~min}$, "intermedio" $5<\mathrm{t}_{\mathrm{EC} 50}<$ a $30 \mathrm{~min}$ y "lento" $\mathrm{t}_{\mathrm{EC} 50}>30 \mathrm{~min}$; de manera que todos los extractos obtenidos en esta investigación se encuentran en la categoría de "intermedio". 
Según el mismo autor, así mismo, la eficiencia anti radical se clasifica así: "baja" EA $\leq 1 \times 10^{-3}$; "intermedia" 1 $\times 10^{-3}<\mathrm{EA} \leq 5 \times 10^{-3}$; "alta" $5 \times 10^{-3}<\mathrm{EA} \leq 10 \times 10^{-3}$ y "muy alta" EA $>10 \times 10^{-3}$, por lo anterior, la eficiencia anti radical de los extractos de papa obtenidos mediante EAU se puede ordenar de mayor a menor así CCQ $<\mathrm{CRM}<\mathrm{PCQ}<\mathrm{PRM}$.

Los resultados de la determinación de ácido clorogénico (Tabla 5), indicaron que la variedad curiquinga presentó mayor contenido en cáscara y pulpa, que la variedad ratona morada. Estos resultados guardan relación directa con los obtenidos para contenido de fenoles totales y actividad antioxidante y coinciden con el hecho de que la mayor concentración de ácidos fenólicos en la papa se encuentran en la cáscara (AlWeshahy et al., 2013). En la figura 2 se presenta uno de los cromatogramas del análisis de ácido clorogénico donde se observa en pico máximo a un tiempo de retención de 2.366 minutos.

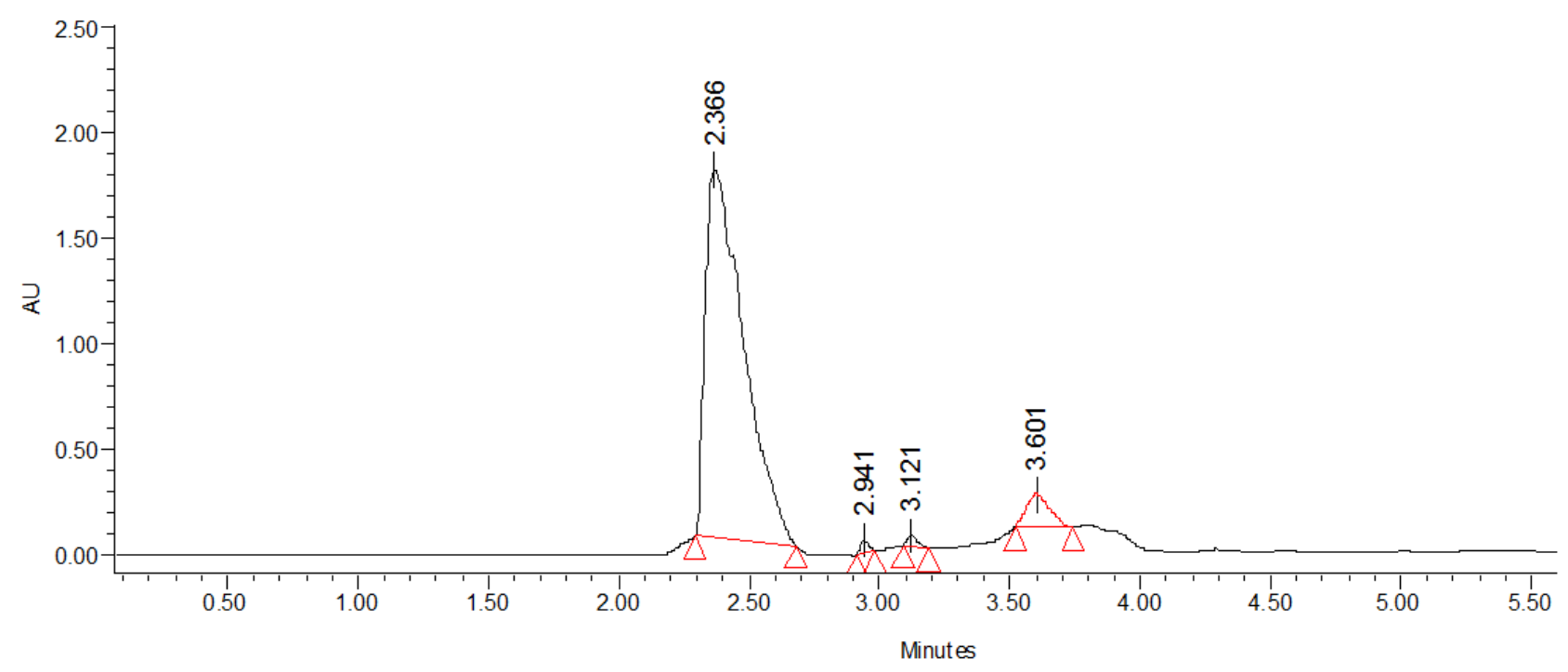

Fig. 2: cromatograma obtenido mediante HPLC para el análisis de ácido clorogénico en cáscara de ratona morada

\section{CONCLUSIONES}

La utilización de ultrasonidos permitió incrementar la eficiencia de extracción de compuestos fenólicos en muestras secas de cáscara y pulpa de papas nativas en comparación con el método de extracción convencional. El tipo de solvente y las condiciones del método de extracción ejercen efectos significativos sobre la cantidad de fenoles totales que pueden ser extraídos de una matriz vegetal y, por consiguiente, sobre los resultados de la evaluación de su actividad antioxidante. Las variedades de papa nativa curiquinga y ratona morada, podrían ser una fuente sostenible de antioxidantes naturales en la dieta de los consumidores.

\section{AGRADECIMIENTOS}

La investigación se realizó en el marco del proyecto "Mejoramiento Tecnológico y Productivo del Sistema Papa en el Departamento de Nariño", financiado por el Sistema General de Regalías FCTel - Gobernación de Nariño, con la colaboración del Grupo de Apoyo a la Investigación y Desarrollo Agroalimentario GAIDA de la Universidad de Nariño.

\section{REFERENCIAS}

Al-Weshahy, A., El-Nokety, M. y otros dos autores, Effect of storage on antioxidant activity of freeze-dried potato peels, https://doi.org/10.1016/j.foodres.2010.12.014, Food Research International, 50(2), 507-512 (2013).

André, C. M., Oufir, M. y otros cinco autores, Influence of environment and genotype on polyphenol compounds and in vitro antioxidant capacity of native Andean potatoes (Solanum tuberosum L.), https://doi.org/10.1016/j.jfca.2008.11.010, Journal of Food Composition and Analysis, 22(6), 517-524 (2009).

Araya, H., Clavijo, C., y Herrera, C., Capacidad antioxidante de frutas y verduras cultivados en Chile, Archivos Latinoamericanos de Nutrición, 56(4), 361-365 (2006).

Boeing, J. S., Barizão, E. y otros cuatro autores, Evaluation of solvent effect on the extraction of phenolic compounds and antioxidant capacities from the berries: application of principal component analysis, Chemistry Central Journal, 8(48), 2-9 (2014).

Brand-Williams, W., Cuvelier, M., y Berset, C., Use of a Free Radical Method to Evaluate Antioxidant Activity, Lebensm. Wiss. u.-Technol, 30(28), 25-30 (1995). 
Campo-Vera, Y., Gélvez-Ordoñez, V., y Ayala-Aponte, A., Ultrasonido en el procesamiento (homogenización, extracción y secado) de alimentos, https://doi.org/10.18684/bsaa(16)102-113, Biotecnología En El Sector Agropecuario y Agroindustrial, 16(1), 102-113 (2018).

Cerón-Lasso, M., Alzate-Arbeláez, A. F. y otros dos autores, Composición Fisicoquímica y Propiedades Antioxidantes de Genotipos Nativos de Papa Criolla (Solanum tuberosum Grupo Phureja), https://doi.org/10.4067/S071807642018000300205, Información Tecnológica, 29(3), 205-216 (2018).

Cuesta-Parra, D.M. y Correa-Mahecha, F, Obtención de fenoles a partir de granos verdes de café, https://doi.org/10.18273/revion.v31n1-2018005, Rev. Ion, 31(1), 31-35 (2018).

Dai, J. y Mumper, R. J., Plant phenolics: extraction, analysis and their antioxidant and anticancer properties. Molecules, 15, 7313-7352 (2010).

Do, Q. D., Angkawijaya, A. E. y otros cinco autores, Effect of extraction solvent on total phenol content, total flavonoid content, and antioxidant activity of Limnophila aromatic, Journal of Food and Drug Analysis, 22, 296-302 (2014).

Guntero, V., Longo, M. y otros tres autores, Comparación de métodos de extracción de polifenoles a partir de residuos de la industria vitivinícola, Asociación Argentina de Ingenieros Químicos, (1), 1-9 (2015).

Kumari, B., Tiwari, B. K y otros tres autores., Ultrasound-assisted extraction of polyphenols from potato peels: Profiling and kinetic modelling, https://doi.org/10.1111/ijfs.13404, International Journal of Food Science and Technology, 14321439 (2017).

Kuskoski, M., Asuero, A. G. y otros tres autores, Aplicación de diversos métodos químicos para determinar actividad antioxidante en pulpa de frutos, Ciênca e Tecnología de Alimentos, 25(4), 726-732 (2005).

Lachman, J., Hamouz, K. y otros tres autores, Differences in phenolic content and antioxidant activity in yellow and purplefleshed potatoes grown in the Czech Republic, PLANT SOIL ENVIRON, 54(1), 1-6 (2008).

Martínez, F., Conservación de agrobiodiversidad andina: El caso de las papas nativas en Colombia, Tomado de Pontificia Universidad Javeriana (2015).

Michiels, J. A., Kevers, C. y otros tres autores, Extraction conditions can greatly influence antioxidant capacity assays in plant food matrices, Food Chemistry, 130(4), 986-993 (2012).

Moreno, E., Ortiz, B. L. y Restrepo, L. P., Contenido total de fenoles y actividad antioxidante de pulpa de seis frutas tropicales, Revista Colombiana de Química, 43(3), 41-48 (2014).

Ola, S., Catia, G. y otros cuatro autores, N. HPLC/DAD/MS characterisation and analysis of flavonoids and cynnamoil derivatives in four Nigerian green-leafy vegetables, https://doi.org/10.1016/j.foodchem.2009.02.013, Food Chemistry, 115(4), 1568-1574 (2009).

Re, R., Pellegrini, N. y otros cuatro autores, C., Antioxidant Activity Applying An Improved ABTS Radical Cation Decolorization Assay, Free Radical Biology \& Medicine, 26(98), 1231-1237 (1999).

Reddivari, L., Miller, J. C. y Hale, A. L., Determination of phenolic content, composition and their contribution to antioxidant activity in specialty potato selections, American Journal of Potato Research, 84(4), 275-282 (2007).

Reyes, L. F., Miller, J. C. y Cisneros-Zevallos, L., Antioxidant capacity, anthocyanins and total phenolics in purple-and redfleshed potato (Solanum tuberosum L.) genotypes, https://doi.org/10.1007/BF02871956, American Journal of Potato Research, 82, 271-277 (2005).

Riciputi, Y., Diaz-de-Cerio, E. y otros 5 autores, Establishment of ultrasound-assisted extraction of phenolic compounds from industrial potato by-products using response surface methodology, https://doi.org/10.1016/j.foodchem.2018.06.154, Food Chemistry, 269 (March), 258-263 (2018).

Sánchez-Moreno, C., Larrauri, J. A. y Saura-Calixto, F., Procedure to Measure the Antiradical Efficiency of Polyphenols, Journal of the Science of Food and Agriculture, 76, 270-276 (1998).

Segura, B., Cadena de valor de papas nativas (Solanum andigenum sp.) en la provincia de Jauja, Perú, Universidad Politécnica de Madrid (2014).

Sharayei, P., Azarpazhooh y otros dos autores, Ultrasound assisted extraction of bioactive compounds from pomegranate (Punica granatum L.) peel, https://doi.org/10.1016/j.Iwt.2018.11.031, Lwt - Food Science and Technology, 101(November 2018), 342-350 (2019).

Solarte-Montúfar, J. G., Díaz-Murangal, A. E. y otros dos autores, D. F., Propiedades Reológicas y Funcionales del Almidón Procedente de Tres Variedades de Papa Criolla, Información Tecnológica, 30(6), en prensa (2019).

Tutunchi, P., Roufegarinejad, L. y otros dos autores, Extraction of red beet extract with $\beta$-cyclodextrin-enhanced ultrasound assisted extraction: A strategy for enhancing the extraction efficacy of bioactive compounds and their stability in food models, https://doi.org/10.1016/j.foodchem.2019.124994, Food Chemistry, 297 (June) (2019).

Villacrés, E., Quilca, N., y otros tres autores. Caracterización física, nutricional y funcional de papas nativas (Solanum tuberosum ssp) para orientar sus usos en Ecuador Physical, nutritional and functional native potatoes (Solanum tuberosum ssp.) characterization to orient their uses in Ecuador, Revista Latinoamericana de La Papa, 15(1), 52-54 (2009).

Wegener, C. B., Jansen, G. y otros dos autores, Special quality traits of coloured potato breeding clones: anthocyanins, soluble phenols and antioxidant capacity, Journal of the Science of Food and Agriculture, 89, 206-215 (2009). 